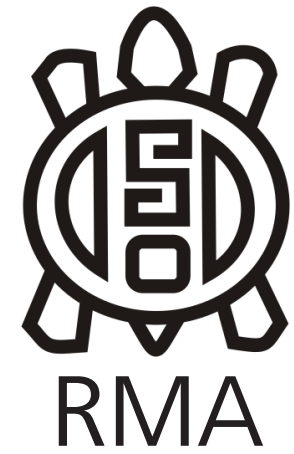

Antropología Social

\title{
La molestia de aliviar el dolor. Etnografía de medicamentos en Cuidados Paliativos Pediátricos
}

\author{
The burden of relieving pain. Ethnography of drug use in Pediatric \\ Palliative Care
}

Candela Rocío Heredia*

* Universidad de Buenos Aires. Facultad de Ciencias Sociales. Instituto de Investigaciones Gino Germani. Buenos Aires, Argentina.

E-mail: candelarheredia@gmail.com

\begin{abstract}
Resumen
La antropología sobre los medicamentos se pregunta por el funcionamiento de la industria farmacológica, la automedicación, la prescripción, la distribución, las explicaciones, eficacias, usos y significados de los medicamentos. Este artículo se inscribe dentro de esta rama de la antropología para escudriñar los sentidos de los medicamentos en un servicio de Cuidados Paliativos Pediátricos de un hospital público pediátrico de la Ciudad Autónoma de Buenos Aires. Para tal fin, se realizó un trabajo de campo etnográfico durante el año 2015. Los efectos negativos y positivos del consumo de medicamentos son dos caras de una misma moneda: son dos polos que se oponen en el interior de pharmakon. A lo largo del artículo se desarrolla la idea que la molestia de aliviar el dolor es inherente a las formas medicamentosas propuestas para el alivio. Se concluye que los medicamentos son entidades en las cuales se condensa ideas y valoraciones sociales y a partir de los cuales las personas buscan ejercer su capacidad de agencia. Además, modelan las sensibilidades y percepciones corporales-afectivas.
\end{abstract}

Palabras clave: Medicamentos; Cuidados Paliativos Pediátricos; Dolor; Efectos adversos; Pharmakon.

\begin{abstract}
Pharmaceutical anthropology tackles the relationship between the pharmacological industry, self-medication, prescription, distribution, uses and meanings of medication. In the context of this anthropological approach, this paper analyzes the meanings related to the drugs used in a pediatric palliative care service of a public hospital in Buenos Aires Capital City. An ethnographic fieldwork was carried out during 2015. It was found that the negative and positive effects of drug consumption are two sides of the same coin: they are two poles opposed inside pharmakon. In this paper supports the idea that the burden of relieving pain is inherent in the usual uses of palliative medication. It is concluded that medications are entities in which ideas and social values are condensed and also that these drugs are used by patients to develop their agency. They model the sensibilities and bodily-affective perceptions.
\end{abstract}

Keywords: Medications; Pediatric Palliative Care; Pain; Adverse effects; Pharmakon.

\section{Introducción}

La utilización de los medicamentos alopáticos se encuentra en crecimiento constante a nivel global, siendo diferentes los sentidos de su utilización de acuerdo a los actores involucrados (que los fabrican, recetan, manipulan, distribuyen o consumen). El trabajo aquí presentado tiene por objetivo una aproximación a los sentidos de los medicamentos en un servicio de Cuidados Paliativos Pediátricos. Para tal fin, se analiza la ambigüedad de la potencia medicamentosa y la agencia en los suministros/ consumos.

El trabajo de campo etnográfico que da soporte a la investigación, cuyos resultados se exponen en este artículo, fue realizado durante el año 2015 en un hospital público pediátrico de la Ciudad Autónoma de Buenos Aires. Se accedió al hospital portando una chaqueta blanca y siguiendo las consultas médicas de profesionales paliativistas (en consultorio y salas de internación). Se siguieron los lineamientos de confidencialidad (todos los nombres que figuran a lo largo del artículo fueron alterados) y consentimiento en la información ${ }^{1}$.

Los Cuidados Paliativos son definidos por la OMS (2002) como "el cuidado total, activo y continuado de pacientes y sus familias cuando la expectativa médica no es la

\footnotetext{
${ }^{1}$ El trabajo de campo etnográfico se realizó concurriendo todos los días al hospital y siguiendo constantemente al equipo médico. Fue parte fundamental para realizar mi tesis de Maestría en Antropología Social (IDES/IDAES-UNSaM) titulada "Sentir dolor. Recorridos, búsquedas y terapias en Cuidados Paliativos Pediátricos" (Heredia, 2017).
} 
curación. Son un enfoque que mejora la calidad de vida de pacientes y familias que se enfrentan a los problemas asociados con enfermedades amenazantes para la vida". Comenzaron a surgir en los años sesenta del siglo pasado principalmente en Inglaterra y Estados Unidos. Se caracterizaron en sus inicios por ser críticos del proceso de medicalización, tecnologización y paternalismo de la medicina (Clark y Semour 1999; Menezes 2004), ponderando el "morir con dignidad" como el propósito de la nueva especialidad médica. Si bien al inicio se volcaron específicamente a personas con cáncer avanzado en el final de sus vidas (los llamados "moribundos"), en el nuevo milenio ampliaron sus límites de competencia hacia personas con enfermedades crónicas. No obstante, el "morir con dignidad"2 sigue siendo un objetivo que se concreta a través del alivio del dolor y garantizando la autonomía de las personas que padecen "enfermedades amenazantes para la vida" (que puedan decidir sobre sus tratamientos, tratos, acompañamiento, lugar de muerte, etcétera).

A pesar que surgieron como críticos a la medicalización y al encarnizamiento terapéutico (la lucha obstinada contra la enfermedad a fin de impedir la muerte), los Cuidados Paliativos presentan un avance de los procesos de medicalización en el final de la vida. Al mismo tiempo, son un intento de "humanización" de la atención. "Humanización" y "medicalización" son "procesos que no deben entenderse necesariamente como opuestos, sino como aspectos complementarios del fenómeno en cuestión" (Alonso, 2013: 2546). Ambos procesos se armonizan, tensionan, se complementan y potencian. La medicalización de los Cuidados Paliativos no tiene un propósito curativo, sino que existe y se expande principalmente para el alivio del dolor y control de otros síntomas (de náuseas, vómitos, disnea, estreñimiento, delirium, sudoración, diarrea, etcétera). En lo que respecta a los Cuidados Paliativos Pediátricos, es la rama abocada a la atención de niños/as y adolescentes ${ }^{3}$. Las implicancias del uso de estos medicamentos en esta rama de la especialidad es lo que se describirá a lo largo del trabajo.

En el primer apartado se presentan las características generales de los medicamentos farmacológicos desde una perspectiva antropológica: como específicos de las sociedades modernas occidentales; como objetos tangibles; objetos de consumo; con poder de transformación; y generadores de bien/mal-estar. En el

\footnotetext{
2 En Cuidados Paliativos ha dejado de utilizarse los términos "muerte digna" o "buen morir" y se habla en los últimos años de "final de vida con dignidad". Cambiaron los términos, pero la filosofía propuesta por Paliativos es la misma.

3 Los Cuidados Paliativos Pediátricos presentan ciertas características propias que los diferencian de los Cuidados Paliativos que se brindan a adultos: existe una mayor sobrevida; muchos diagnósticos son poco frecuentes y sólo se dan en la niñez; muchas enfermedades son genéticas, por lo que puede haber más de un/a niña/o afectada/o por familia; se prioriza la continuidad del proceso educativo (que continúen o inicien en las escuelas) y el juego (Salas, 2004).
}

segundo apartado describo y analizo etnográficamente los "efectos adversos" de los fármacos indicados por los médicos paliativistas. En el tercero ahondo en la manera en que adolescentes se autosuministran medicamentos y contrarrestan los efectos no deseados. Por último, concluyo con algunas reflexiones sobre el valor político de los medicamentos.

\section{Antropología sobre los medicamentos}

La antropología sobre los medicamentos surgió en los años ochenta, interesada en el funcionamiento de la industria farmacológica, la automedicación, la prescripción, la distribución, las explicaciones, eficacias, usos y significados de los medicamentos. No resignarse a entender a los medicamentos como meras sustancias químicas y observar las dinámicas en que son comprendidos es el principio de esta área de estudio.

Enfermar es un universal en la experiencia humana y cada sociedad desarrolló las formas de tratar ese enfermar (qué es enfermar, sus causas, soluciones, prevención y alivio está determinado culturalmente por cada sociedad específica). Por esta razón, los sujetos se construyen, en parte, dentro de los procesos de padecimiento y respuesta a ellos. Esos procesos son sociales y allí se establece colectivamente la subjetividad. "La respuesta social a la incidencia de enfermedad, daños y/o padecimientos (...) constituye una estructura necesaria para la producción y reproducción de cualquier sociedad" (Menéndez, 1994:71). Todos los conjuntos sociales generarán conocimiento para erradicar los padecimientos. Para el caso de las sociedades modernas occidentales, su característica central en la forma de sanar ha sido la implementación masiva de sustancias llamadas "medicamentos". Los medicamentos fabricados por la industria farmacéutica tienen la particularidad de, como fenómeno de la expansión del capitalismo a escala mundial, encontrarse en distintos puntos del planetario y permear diversas sociedades; numerosas investigaciones dan cuenta de cómo aparecen y circulan los medicamentos en sociedades indígenas y rurales (Hardon 1987; Lagndon 1988; Etkin 1992; Kamat y Nichter 1998; Drovetta 2009; Diehl y Grassi, 2010; Cuyul, Rovetto y Specogna 2011; Lorenzetti 2012; Cebolla Badie, Dell'Arciprete, Fontes, Hirsch y Orlando 2013; Cantore 2014; Hirsch y Lorenzetti 2016). Al mismo tiempo crecen las etnografías que analizan la dinámica del comercio farmacéutico internacional entrelazado con las desigualdades globales y reforzado por una medicina lucrativa (Petryna, Kleinman, Lakoff 2006). La antropología sobre los medicamentos está interesada en observar cómo se mueven y significan los medicamentos en estas múltiples sociedades ${ }^{4}$, en sus instituciones y cómo impacta en las subjetividades.

\footnotetext{
4 Por supuesto que la utilización de medicamentos no es ni exclusiva de las sociedades moderno occidentales ni total en el planetario. La pluralidad médica y complementariedad terapéutica es frecuente (aun cuando no implique la utilización de fármacos biomédicos)
} 
Siguiendo la literatura de esta área de estudio, podemos sintetizar algunos atributos de los medicamentos. En primer lugar, debemos decir que (1) los medicamentos son objetos concretos. En tanto objetos concretos, pueden intercambiarse y objetivan significados en ese intercambio. Además (2) son símbolos y muestra de esperanza para las personas que esperan mejorar su salud. Esto es así ya que los medicamentos (3) tienen principalmente el poder de transformar. Se les atribuye este poder en la medida que se espera que modifiquen el cuerpo de quien los consume. $Y$ he ahí su gran valor: los medicamentos producen transformación. Y lo hacen en tanto pueden erradicar la enfermedad del cuerpo (como los antibióticos ante una infección bacteriana) o tornar el cuerpo débil en cuerpo fuerte para disminuir el riesgo de contraer la enfermedad (a través de vitaminas, tónicos y vacunas) (Persson 2004; Van Der Geest, Whyte y Hardon 2002).

Pero los medicamentos no se utilizan sólo cuando se trata de enfermedad, sino que acompañan procesos vitales y aparecen así en el parto de las mujeres, en la lactancia, en la menopausia; son llamados a actuar para cuestiones estéticas: hacer algo por la calvicie, el acné, reducir estrías, arrugas, aumentar o disminuir el peso, etcétera. Porque además de sustancias terapéuticas son (4) mecanismos socializadores de las formas en que pensamos el "bienestar" más allá de la salud y la enfermedad (Williams, Martin y Gabe, 2011). Los medicamentos son entonces (5) un objeto de consumo (Lopes 2001). Se consumen los medicamentos con la esperanza de transformar y generar bienestar.

Colaboran en crear una esperanza para alcanzar una imagen o estilo de vida valorado positivamente por la sociedad. Para Rose (2012), "la política vital de nuestro siglo no se encuentra delimitada por los polos de la salud y la enfermedad, ni se centra en eliminar patologías para proteger el destino de la nación. Antes bien, se ocupa de nuestra capacidad, cada día mayor, de controlar, administrar, modificar, redefinir y modular las propias capacidades vitales de los seres humanos en cuanto criaturas vivas" (p. 25). Es decir, las tecnologías médicas contemporáneas se ocupan del control de los procesos vitales, diluyéndose la demarcación entre lo enfermo y lo sano, y entre lo terapéutico y lo comercial. Los medicamentos, como una tecnología médica, actúan modificando las capacidades vitales a fin de acercar a los sujetos a los ideales estéticos y de bienestar que han construido (Van Der Geest, Whyte y Hardon 2002).

Pero el uso de medicamentos no lleva a la inevitable y pura transformación beneficiosa de los cuerpos. Los medicamentos generan malestar y bienestar simultáneamente. De allí la importancia de suministrarlos en determinadas dosis, dejarlos fuera del alcance de los niños, utilizar protectores gástricos (como el omeprazol) para poder consumir otros medicamentos y advertir sobre los efectos secundarios de su uso. Incluso existen dosis tóxicas para el suicidio (Van Der Geest, Whyte y Hardon 2002). A lo largo de este artículo describiré esa ambigüedad medicamentosa (de generar bien/mal-estar) y la agencia en los suministros/consumos de medicamentos en un servicio de Cuidados Paliativos Pediátricos.

\section{Incomodidades de los fármacos}

La ambigüedad medicamentosa refiere a la simultánea producción de bien y mal-estar que producen los fármacos. Esto se refleja en el mismo origen de la palabra "fármaco", derivada del griego pharmakon, que significa tanto "remedio" (medicina o cura) como "veneno" (toxico). Y a pesar que pharmakon siempre alberga dentro de sí la complicidad de estos valores contrarios, en la medicina moderna se invisibiliza uno de sus polos y se erige tan sólo uno, el más tranquilizar de ellos, el "remedio". De esta forma, la virtud de ser "remedio" enuncia el triunfo del conocimiento occidental, racional, científico, técnico y terapéutico. El proceso de medicalización que continúa en expansión hace que los sujetos comiencen a explicar y atender sus padecimientos a través de técnicas y concepciones biomédicas (Menéndez, 2003). En la medicina moderna (o biomedicina) se enaltece el "remedio" como solución a los problemas del conjunto social. Y el lado oscuro de la misma medicina, por su parte, es ocultado y olvidado. No obstante, las investigaciones antropológicas sobre los procesos de salud-enfermedad-atención-cuidados (donde hay utilización de fármacos) vuelven a revelar la ambigüedad medicamentosa (Persson 2004).

En los Cuidados Paliativos Pediátricos (CPP) se utilizan fármacos para aliviar el dolor. Estos fármacos son analgésicos y opioides. Los analgésicos son AINESs (drogas antiinflamatorias no esteroideas -como el ibuprofeno, naproxeno, keterolaco o diclofenaco) y el paracetamol. Los opioides son la codeína, tramadol, matadona, fentanilo o morfina (entre otros). A su vez, se utilizan co-adyuvantes a estos fármacos, que son otros fármacos que ayudan a disminuir aún más el dolor o a minimizar los efectos adversos de las medicaciones ya suministradas. Estos co-adyuvantes a los analgésicos o a los opioides son los anticonvulsivantes, los laxantes, antieméticos y los antidepresivos. Las vías de administración de todos estos fármacos son en general la ingesta oral, administración subcutánea e inyección intravenosa (Vega 2016). La utilización de esta variedad de compuestos químicos tiene implicancias consideradas negativas (a pesar de su propósito de alivio).

Durante mi trabajo de campo, he observado que la morfina es el fármaco más utilizado por el equipo de CPP. La amargura de la morfina es una de las tantas molestias que ocasiona, la cual fue manifestada en una visita a Lautaro, un pequeño de nueve años. 
A Lautaro le habían diagnosticado leucemia, tenía una mucositis oral y estaba internado en la sala de Infectología. Entramos con Victoria, médica de CPP, a su habitación. Apenas nos vio, el niño cerró los ojos, dio media vuelta y se cubrió con las sábanas. Nos sentamos a su lado y Victoria comenzó a hablarle mientras lo acariciaba.

-Hola, Lauti -lo saludó con voz tierna-. Nosotras somos de paliativos. Nos dijeron que estabas con un poco de dolor y por eso venimos ¿Nos querés decir dónde te duele? Así te ayudamos a no tener más dolor...

Lautaro no se movía. Victoria siguió acariciándolo en silencio. Luego de unos veinte minutos el niño destapó su rostro y la miró. Victoria sonrió con ternura. Siguió acariciándolo y le preguntó:

- ¿Me querés mostrar cómo está la boca?

Lautaro negó con la cabeza.

-No te voy a hacer nada. Sólo voy a mirar un poquito.

Lautaro volvió a negar con la cabeza.

-Bueno, otro día venimos y, si querés, nos mostrás cómo está la boca, ¿dale?

Lautaro afirmó con la cabeza.

-Ahora te vamos a indicar un poco de morfina para que tomes, ¿sabés? -prosiguió Victoria con voz suave, sin dejar de acariciarlo.

Lautaro negó una vez más con la cabeza.

- ¿No querés probar un poco? Dale, probá tomarla aunque sea una vez. Y si no te gusta, vemos que te la den de otra forma, que no sea por la boca.

El niño continuaba negando con la cabeza.

-Una sola vez probá. Después de tomarla, podés tomar algo rico como un jugo -insistió Victoria, pero el pequeño no cedía-. Sé que es fea la morfina, que es amarga, pero te va hacer bien. La tomás y en seguida tomás algo dulce y rico que te guste.

Sin consenso en la toma de morfina, nos despedimos de Lautaro con la promesa de volver más tarde. Salimos de la habitación. En el pasillo nos encontramos con un médico de la sala y Victoria le dijo:

- Hablamos con Lautaro, pero no quería tomar la morfina. Yo prefiero que sea vía oral para que no lo pinchen. Pero no la quiere tomar. Pobrecito. Después volvemos a ver si quiere tomarla... Pero es entendible que no quiera, es súper amarga.

(Diario de campo, 20/08/15)

A pesar de los intentos de Victoria, Lautaro no quiso probar $^{5}$ la morfina. La morfina es súper amarga si se

\footnotetext{
${ }^{5}$ Utilizo itálicas para señalar el origen nativo de las palabras, remitiendo por ello al sentido que los propios actores le dan a las mismas, o
}

ingiere por la boca. Pero la otra opción que Victoria veía posible era por otra vía: que lo pinchen. Dentro de ese contexto, donde el objetivo es aliviar el dolor y donde el medio para hacerlo es la morfina, se produce un movimiento donde el fin es la morfina, y el medio puede ser la ingesta oral o ser pinchado. Pobrecito, dice Victoria, palabra que parecería evidenciar una valoración negativa a cualquiera de los medios. Pero se erige el carácter de inevitabilidad de los procedimientos médicos.

Para los Cuidados Paliativos, minimizar el dolor es otorgar mejor calidad de vida, por esta razón es que trabajan con analgésicos y opioides. Éstos son fármacos que, se asegura desde esta especialidad, pueden producir bienestar en tanto tienen el poder de, utilizados de manera experta, aliviar el dolor. Y este alivio tiene inherentemente acciones molestas para los/as niños/as que son reconocidas por los/as médicos/as, pero necesarias.

En la misma semana que fuimos a ver Lautaro, recuerdo que fuimos con Victoria a ver a Joaquín.

-Estoy contento. Ahora que no estoy más con morfina, puedo ir bien al baño -dijo Joaquín. Joaquín era un adolescente. Le habían amputado el pie: tenía un contra sarcoma ubicado allí. La primera vez que lo vi, unos meses atrás, Victoria le miró las pupilas y acotó "a este chico le está sobrando morfina". Ajustó la dosis y transcurridos unos meses dejaron de suministrársela.

Joaquín estaba internado en Clínica, acostado en una cama. Entramos con la médica a su habitación.

-¿Cómo estás Joaquín? -preguntó Victoria.

-Estoy contento. Ahora que no estoy más con morfina puedo ir bien al baño.

-Sí, la morfina constipa; por eso siempre indicamos un laxante cuando indicamos morfina.

-Sí, pero igual me cuesta ir al baño con la morfina.

- ¿Y ahora vas bien?

-Sí. No pude dormir mucho porque me despertaron a las siete de la mañana para pincharme.

- ¿Te sacaron sangre?

-Sí.

-Bueno, son necesarios los análisis para saber bien cómo estás... Pero sí, es molesto que te despierten tan temprano para pincharte...

-Sí. Pero bueno, por lo menos puedo ir al baño ya. Voy todos los días.

-Qué bueno. Nos pone contentas verte mejor y verte contento. Y sí, también es molesta la morfina, pero fue necesaria para que no tengas dolor.

(Diario de campo, 23/08/15)

\section{La constipación es otro de los efectos adversos}

bien indicando su procedencia. 
reconocidos tanto por Victoria como por Joaquín. Dicho efecto intenta apaciguarse presentando la medicación en una díada: morfina-laxante. Sí, pero igual me cuesta ir al baño con la morfina: díada que no siempre es eficaz.

Los procedimientos médicos, como son los análisis de sangre regulares en personas hospitalizadas, se constituyen en molestias con las cuales -tanto médicas/os como familiares y personas internadas- lidian a diario. Son necesarios. Son una forma de comunicación, una forma de conocer y una forma de saber dentro del régimen de verdad-autoridad biomédico. Es la mirada clínica a través de la cual se interroga al cuerpo. En estas prácticas de tecnología se describe el estado de una persona a través de la cuantificación y clasificación de células, hormonas, proteínas, etcétera. Se realizan con el objetivo de saber bien cómo estás.

Existe una reorganización biomédica de las experiencias subjetivas de enfermar y padecer en donde la enfermedad es ubicada como un observable, algo "real" (Persson 2004:50). Los Cuidados Paliativos colocan el dolor como "algo real", que puede comprenderse con la progresión o remisión de las células cancerígenas, de las infecciones, etcétera, que los análisis y la inspección clínica pueden objetivar. Es en el espacio físico del cuerpo donde se ubica el dolor que debe aliviarse.

El análisis corporal crea objetos tangibles, órganos palpables y vías internas que facilitan la aplicación de las terapias. Éstas, al igual que los cuerpos y sus partes, son interpretadas como sustancias concretas. "La sustancia administrada señala el objetivo identificado $y$, a través de alguna interacción fisicoquímica (por ejemplo, entre una molécula de fármaco y una célula humana), corrige lo que la biomedicina ha determinado como anormal y, por lo tanto, restablece la normalidad corporal" (Persson, 2004:50). Para los Cuidados Paliativos, la anormalidad que debe corregir es la presencia de dolor. El dolor es un síntoma de la enfermedad amenazante para la vida. "Si el problema es físico, entones el remedio debe ser físico. Los medicamentos aparecen como respuesta perfecta para el problema" (Van Der Geest y Whyte, 2011: 465). Si el dolor es físico, los analgésicos, opioides y sus co-adyuvantes son las sustancias físicas que aliviarán ese dolor.

Otro joven en CPP era Luis, de dieciséis años y con un tumor en su pierna. Estaba con morfina y amitriptilina (un antidepresivo tricíclico).

-Me pasa que tengo muchas ganas de orinar, y cuando voy al baño, no puedo. Me cuesta-dijo Luis.

-Eso es un efecto adverso de la medicación que te estamos dando para el dolor. Vamos a bajar entonces la amitriptilina -dijo Francisco, médico de CPP. Luego miró a Agostina, la hermana de Luis, que era médica pediatra de otro hospital, y procedió a explicarle en términos médicos el motivo por el cual la amitriptilina acarreaba problemas urinarios. (Diario de campo, 17/05/15)

Y Cristóbal, otro niño de once años de edad también sufría efectos al intentar aliviar su dolor.

-Cristóbal no ve bien -nos anunció por entonces una médica de Traumatología.

- ¿Ve mal? Entonces debe ser el gabapentin que le indicamos para el miembro fantasma -le contestó Victoria.

Cristóbal seguía internado en la Unidad de Traumatología y comenzó a ver raro.

-Consultó la psicóloga que habla con él -aclaró la médica de Traumatología-; Cristóbal le dijo que veía raro y a ella le parecía que no era... algo psicológico, digamos. Quería saber si era algo de la medicación ¿Entonces vos decís que es el gabapentin?

-Sí, yo creo que es lo que le damos para el dolor del miembro fantasma. Ahora le ajustamos la dosis y vamos a hablar con Cristóbal.

-Dale, yo le aviso a la psicóloga.

(Diario de campo, 10/12/15)

Ver raro, puede formar parte de estos efectos adversos ¿A qué se debe que vea raro? O mejor ¿a quién le compete responder esa pregunta? La psicóloga de Cristóbal desechó la posibilidad que el problema pertenezca al campo de su disciplina, motivo por el cual no fue enunciado-intervenido desde la Psicología. Victoria tomo para sí el problema, definiéndolo y planteando una acción -Ahora le justamos la dosis-.

El poder de transformación de los medicamentos se expande en los cuerpos, en las relaciones, situaciones y modos de comprensión de los sujetos. Por lo tanto, los lenguajes y modos de tratar son afectados por los medicamentos. Para el caso del lenguaje médico, se puede sostener que tiene un papel de conductor de la medicación y de la enfermedad hacia el terreno de la concreción. En el terreno de lo concreto se ubica tanto la enfermedad, el dolor, como la medicación. El lenguaje médico que institucionaliza el diagnóstico y tratamiento da eficacia simbólica a los medicamentos.

"En su carne sufriente [del sujeto que enferma], viene a incorporarse el diagnóstico médico, hiriendo al sujeto en el mismo acto que abre el campo de su existencia; hiriéndolo porque el significante violenta el cuerpo y remolca significados ominosos; también porque, mediando el diagnóstico, la medicina irrumpe en el cuerpo así constituido con la parafernalia del tratamiento" (Reyes Pérez, 2011:206).

El significante -Eso [que te sucede, que ocurre en tu 
cuerpo], es un efecto adverso de la medicación que te estamos dando para el dolor- pone en evidencia que los intentos de alivio al dolor no son gratuitos. Acarrean efectos en el cuerpo. Desde un saber-poder se nomina y desde ese lugar se explica. En este caso, fue a través de un lenguaje médico que Francisco explicó a Agostina (médica pediatra y hermana de Luis) los pormenores del proceso. Los términos eran tan ajenos a mí que no conseguí registrar ni entender cómo es que, desde la biología y la anatomía, determinadas dosis de amitriptilina causan problemas urinarios. Pero lo que observé en esa performatividad de la consulta médica era la enunciación de carácter constatativa (que reafirma el diagnóstico en términos anotomopatológicos). Lo que sucedía en el cuerpo de Luis al tiempo que se definía, explicaba y brindaba pautas a seguir desde un discurso médico, ponía de manifiesto (con el mismo lenguaje), los efectos de la enunciación-intervención médica. "La enfermedad adquiere por sí sola su propia sustancia dentro del cuerpo de la persona. El médico da forma y la define para el paciente" (Illich 1975: 152). En el cuerpo de Luis, de Cristóbal, Joaquín y tantos otros niños/as y adolescentes, está el problema, el efecto adverso. Los médicos/as en el mismo acto de verbalización, lo ubicaban allí, lo conceptualizaban, explicaban y proponían, cuando podían, una solución medicamentosa, que no era otra cosa que el ajuste de dosis, una disminución de (ambos polos de) pharmakon. Porque si bien la biomedicina sólo llama efecto adverso al polo oscuro de pharmakon, el alivio del dolor también lo es. Ambos son efectos, mutuamente adversos, en el sentido más etimológico del término. "Adverso" proviene del latín adversus que significa "que está al frente" o "que se opone". Tanto el alivio como la molestia se oponen mutuamente en la terapia medicamentosa de los Cuidados Paliativos. Lo positivo y lo negativo de los medicamentos para el dolor son dos caras de una misma moneda. Disminuir una dosis es disminuir los efectos positivos-negativos.

\section{Poder de prescripción y agencia corporal}

Una mañana del año 2015, fuimos con María, médica de Cuidados Paliativos Pediátricos, al sector de Oncología. Allí estaba Santiago con sus abuelos. A Santiago le habían amputado la pierna derecha por un tumor que tuvo tiempo atrás. Ahora, con 18 años, usaba muletas, le faltaba el pelo sobre su cabeza, en las cejas y pestañas. Le habían suspendido la quimioterapia ya que el equipo médico prefería realizar unos estudios antes de recomenzarla...

-Con el tema del dolor y las medicaciones, ¿cómo estás? - le preguntó María.

-Sigo con mucho dolor acá -dijo Santiago señalando el tórax y agregó-. Quería ver de empezar a tomar morfina.

-Está bien. Vos estás con metadona ahora -dijo mirando su historia clínica-. Habría que rotar a morfina. Se puede, el tema de la morfina es que viene líquida, no en comprimidos.

- Yo no puedo tomar nada líquido -contestó Santi-. No puedo tomar ninguna medicación líquida. Me da arcadas.

- Ya lo sabemos, por eso te aclaro. En la farmacia de acá del hospital, la morfina que se da es líquida. Existe en comprimidos, pero la tenés que comprar afuera y sale plata.

-No tenemos plata para comprarla en comprimidos... Bueno, puedo intentar tomarla. -Ese es el problema; que no se trata de intentar con morfina líquida y si no funciona, volvés a la metadona. Pasar de metadona a morfina no es fácil, hay que ir ajustando las dosis y demora unos días. Y si intentás tomar la morfina líquida y no podés, vas a estar con dolor... ¿Qué querés hacer?

-Bueno, sigo con la metadona entonces.

-Está bien. Y Santi, ¿a la noche cómo estás durmiendo? ¿Podés dormir bien?, ¿o te despertás por el dolor?

-Duermo bien.

- ¿Con cuánto estabas de clonazepam? preguntó María mirando la historia clínica.

-... y tomo dos de clonazepam para dormir. -i¿Dos?! -preguntó sorprendida.

-Sí, ustedes me indicaron el clonazepam.

-Nosotros te indicamos medio, no dos.

-Puede ser...

Se hizo un silencio en donde Santiago y María intercambiaron miradas.

-El clonazepam en esa dosis te lo indicaste vos -sentenció María.

(Diario de campo, 15/11/15)

Santiago tomaba clonazepam de una forma que no era la indicada por la expertise de las/os médicas/os. En primer lugar, esto es posible por una delegación previa que ha realizado la propia expertise hacia el joven: es él quien debe responsabilizarse de tomar sus medicaciones en su hogar y hacerlo de la manera "indicada". Esta delegación podría leerse como una práctica de autocuidado que se exige al seguir los tratamientos de manera ambulatoria. Quienes están internados, tienen márgenes más estrechos de elección: enfermería se encargará de pasar las medicaciones a través de sondas, vías, catéteres, se encargará de ofrecerla en bandejas metálicas y controlar su ingesta, estando el sujeto en una cama; sujeto que en estos casos es bebé, niña/o o adolescente, lo que profundiza las asimetrías en la relación y arrasa con los márgenes de elección. Pero quienes están en sus hogares y son más grandes, se les exige obediencia, adherencia; en suma, autocuidado. El autocuidado ha sido definido como "los modos en que la práctica médica ya no concentra su poder solo en la institución, sino que ejerce influencia en otros ámbitos y espacios de la vida relacionados con condiciones íntimas y personales de los pacientes" (Del 
Monaco 2013:70). Automedicarse siguiendo los cánones de los/as médicos/as es considerado autocuidado desde la perspectiva biomédica. Seguir fielmente las indicaciones se constituye en un requisito para el estar bien, para ser un "enfermo saludable" (Dominguez Mon 2015); autocuidado que tiene un fuerte componente moral al ser regulado por una moralidad dominante que guía las pautas de lo que es saludable o no, que establece el control de sí y la buena presencia en una constante interacción del sí mismo con el "sí mismo social" (Bates en Del Monaco 2013).

Pero en la medida en que los dispositivos de poder médico(s) intentan de manera capilar tomar para sí otros dominios de la vida cotidiana, los agentes toman para sí, elementos de la autoridad médica. Es decir, al abrirse la posibilidad de consumir la medicación en un ámbito más allá del hospital, se abre la posibilidad de hacerlo según los propios criterios (motivo y cantidades). Durante mi trabajo de campo he observado numerosos adolescentes que manejaban ellos/as mismos/as el consumo de clorazepam, ibuprofeno, distintos analgésicos y antiinflamatorios.

Si bien dentro del hospital pueden negociarse una u otra medicación (metadona o morfina, por ejemplo), en la casa, Santiago era quien se automedicaba (al igual que otros jóvenes). Tomó para sí ese elemento tan preciado del saber y la autoridad médica que es la gestión del bienestar mediante los medicamentos.

"Los medicamentos son sustancias que tienen la capacidad de transformar a los organismos vivos y mejorar el estado de salud de las personas. A partir de ellos, se construyen percepciones en torno a las enfermedades, a su eficacia para curar y al papel de los profesionales de la salud" (Cebolla Badie et al, 2013). La apropiación y uso de los medicamentos por parte de estos jóvenes, habla de cómo las prácticas de automedicación se imbrican en las nociones de bienestar, y cómo se distribuyen las labores en los tratamientos. Los medicamentos objetivan el arte de aliviar de los/as médicos porque "a través de los medicamentos, las personas gozan de la expertise médica sin la incomodidad de realmente haber ido al médico" (Van Der Geest y Whyte 2011: 468). Los medicamentos tienen el poder de transformar y la ciencia médica acredita su poder. Y esos medicamentos allí están, disponibles para que un joven en cuidados paliativos los use. $Y$ al usarlos según su propio criterio, quiebra en parte la hegemonía de los profesionales y muestra su agencia para manipular los medicamentos ${ }^{6}$.

El cuerpo que es transformado por la ingesta de fármacos es un cuerpo vívido que agencia continuamente. $Y$ ese

\footnotetext{
6 Por supuesto, dichos criterios se crean en gran parte durante los itinerarios terapéuticos, por lo que en los propios criterios está la marca de la doctrina médica. A pesar de esto, los jóvenes trastocan la autoridad y usan los medicamentos desafiando las dosis y los tiempos indicados.
}

cuerpo no sólo interactúa con los fármacos, sino que los cuerpos "interactúan de forma más compleja con otras materialidades, como los alimentos que ingiere y degusta" (Rohden 2018: 221). Y en esas múltiples interacciones podemos seguir indagando en los movimientos creativos que realizan los jóvenes para lidiar con los fármacos y sus efectos ambiguos. Tal fue el caso de una joven, Pilar de quince años. Una mañana concurrió la joven, con su madre, al consultorio de Paliativos. La recibió la pediatra María. Le preguntó cómo estaba. Pilar tenía un diagnóstico médico de vasculitis (enfermedad autoinmune que produce inflamación en los vasos sanguíneos). Consultaba regularmente con el equipo por cefalea y dolor neuropático provocado por la enfermedad. Pilar contestó que estaba bien. No tenía dolor.

-Estás muy bien -concluyó María.

-Sí. Estoy bien. El otro día me desmayé en el colegio, pero estoy bien-dijo Pilar.

-i¿Cómo que te desmayaste?!

-Sí. Me bajó la presión -expresó riendo.

Seria, María agregó:

-Bueno, eso es un tema. Tenemos que ver si no es un tema de la medicación. Tenemos que consultar con el cardiólogo y avisar a Reumato[logía]. Hay que revisar todas las medicaciones con las que estás ¿Ahora vas al cardiólogo? Te hago una notita para que le entregues así sabe ¿Te desmayaste una o varias veces?

-Sí, voy ahora porque me tengo que hacer un electro[cardiograma]...-contestó suspirando y luego dijo tímidamente- Fueron dos veces que me desmayé...

-Bueno, tomá la notita. Se la entregás al cardiólogo y cuando vayas a Reumato también le avisás. Todos tenemos que revisar las medicaciones -dijo Victoria con tono de preocupación.

-Bueno -contestó Pilar tomando la nota entre sus manos y agregó-. Aunque también puede ser por otra cosa.

- ¿Por qué cosa?

-Tal vez sea porque no como.

-¿No comés?

-No, porque estoy gorda.

- ¿Cómo es eso?

-Me siento gorda y por eso no como. Las veces que me desmayé en el colegio no había desayunado y no había comido nada. Estoy como obsesionada; si como algo me quedo pensando todo el tiempo que no debía haberlo comido. Me siento gorda. La medicación que me dan hace que sea gorda. Yo no quiero ser gorda.

-Son necesarios los corticoides, Pili.

-No los quiero tomar -dijo agachando la mirada. 
-Te hacen bien, ya tenés la mano bien, no te duele, no te duele la cabeza y sos hermosa. -Gracias.

-Bueno, te damos un turno para vernos el mes que viene ¿Te parece?

(Diario de campo, 04/09/15)

Si "el cuerpo enfermo, con dolor, adquiere nuevos sentidos, interpela y es interrogado de forma renovada" (Alonso 2009: 108), la materialidad del cuerpo constituye un aspecto central como expresión de la propia medicación para aliviar ese dolor, para tratar a ese cuerpo enfermo. En la contemporaneidad, el cuerpo es expresión de una ambigüedad: es objeto de control y atención, y al mismo tiempo, resultante de incertidumbre, angustia y malestar; fenómeno relacionado con la búsqueda del cuerpo perfecto a partir de un patrón de belleza reproducido por el universo mediático, que lleva a la mutación constante del cuerpo, el cual es visto como una entidad a ser trabajada y donde se establece una relación entre delgadez, sexualidad y feminidad. Las estrategias de no comer que llevaba a cabo Pilar pueden ser leídas como una recuperación de "una dimensión de lo vívido corporal", de personalización del cuerpo, donde su agencia va en dirección a una modificación e intervención en función de sus valores. No es entonces ni un problema de salud mental ni el resultado de modismos como un elemento constitutivo de la sociedad de consumo (Ortega en Oliveira 2012). Es el cuerpo vívido (Kottow y Bustos 2005) el que se hace presente en esas decisiones; decisiones que en el ámbito biomédico de la salud suele verse como desvío, riesgo, algo serio, que debe evaluarse, que debe acompañarse (controlarse) por especialistas en sus sistemas expertos.

Para Good (1994), los padecimientos implican un quiebre y una deconstrucción del mundo. Pero también las experiencias de enfermedad y el dolor implican acción, un movimiento donde se revela la potencialidad del actor. $Y$ es en este acto de construcción donde aparece la agencia, las formas de sentir y percibir, las cuales revelan a su vez, mecanismos más complejos de interrelaciones. La transformación por la medicación produce bienestar. Pero simultáneamente produce malestar. El precio por no tener dolor, parecería ser la hinchazón por corticoides. Sin embargo, ese no es el punto de llegada, sino el continuum del cambio corporal en sus múltiples interrelaciones. Pilar contrarrestaba la hinchazón por corticoides, con la práctica de dejar de comer. Y el verse o sentirse "gorda", no se relaciona exclusivamente con el uso de corticoides. Si bien para María era problemático e inevitable el consumo de la medicación indicada y Pilar tenía razón, los corticoides la hinchan, "las maneras de sentir forman parte de los entramados mayores que limitan, a la vez que producen, modalidades posibles de sentir(se), de ser sentidos, de percibir(se) y de ser percibidos" (Candil 2015: 64)

\section{Reflexiones finales}

Los medicamentos modelan las sensibilidades y percepciones corporales-afectivas, "son productos de la cultura humana, pero también productores de la misma, que afectan el sentido del ser y la vida social de las personas" (Persson 2004:46). A diferencia de otras medicinas que deben ser preparadas antes de ser aplicadas, los medicamentos que utilizan en Cuidados Paliativos, ya están prontos para ser utilizados. La adivinación, las ceremonias colectivas, el sacrificio, la preparación de plantas, colocan a la persona que padece en manos de otras personas (curanderos, líderes religiosos, etcétera). Los medicamentos, en cambio "le permiten asumir, en sus propias manos, su condición" (Van Der Geest y Whyte 2011:461) y objetivan el poder científico-médico. Los analgésicos y antiinflamatorios están al alcance de jóvenes y ellos/as los utilizan según sus criterios, pero asignándoles el poder de transformación. Son consumidos en la medida que tienen una eficacia terapéutica: ayudan a estar mejor. Hay una ciencia médica que acredita ese poder, una industria farmacéutica que los produce y un cuerpo médico que los prescribe. Pero también hay jóvenes que toman para sí ese poder y lo movilizan según sus propios intereses. Se cuestiona entonces la pauta médica de quien los prescribió, pero la creencia de transformación sigue intacta. La autoridad responsable que señala el uso de los mismos es el médico. Pero los/as jóvenes en tratamiento ambulatorio son quienes gestionan su prescripción y consumo. En cualquiera de los casos, continúan siendo símbolos y muestra de esperanza que algo cambiará a partir de su ingesta.

Las modificaciones que producen los medicamentos no son sólo en dirección a una mejora. También posee consecuencias negativas. Ambas consecuencias son reconocidas por todos los actores: la industria coloca en los prospectos los "efectos adversos", los médicos saben identificar qué problema en el cuerpo es un "efecto adverso" de la medicación y las personas que los consumen, encarnan dicho efecto. Las personas lo manifiestan, se quejan y hasta llegan a hacer algo para contrarrestar ese efecto. Los medicamentos reconfiguran los cuerpos. Y los cuerpos vívidos, a su vez, reconfiguran los efectos de los medicamentos en un mundo de entramados mayores que también condicionan las formas de percepción de dichos efectos.

Como se señaló anteriormente, los efectos negativos y positivos del consumo de medicamentos son dos caras de una misma moneda: son dos polos que se oponen en el interior de pharmakon. La molestia de aliviar el dolor es inherente a las formas medicamentosas propuestas para el alivio.

Las capacidades vitales de los seres humanos están siendo moduladas por los medicamentos. En el hospital donde 
se realizó el trabajo de campo etnográfico, los modos de sentir y percibir confort o disconfort están íntimamente ligados a la administración de medicamentos. El control y la definición del dolor en niños/as y adolescentes en Cuidados Paliativos se reorganizan en la interacción medicamentosa.

Los medicamentos tienen importancia económica y valor político. No solamente tienen importancia económica por el hecho de que haya una industria que los fabrica con ganancias millonarias, con estrategias de marketing en los medios masivos de comunicación y subvenciones a especialidades médicas (auspicio de congresos y financiamiento a investigaciones farmacológicas). Sino que, observando los circuitos económicos de los medicamentos, se trasluce la dinámica de atención a la salud, con sus desigualdades de acceso. Y el valor político está en su capacidad de reconfigurar corporalidades y padecimientos. Los medicamentos son entidades en las cuales se condensa ideas y valoraciones sociales y a partir de los cuales las personas buscan ejercer su capacidad de agencia.

\section{Agradecimientos}

Este trabajo es parte de mi tesis de Maestría en Antropología Social (IDES/IDAES-UNSaM) y fue reelaborado en el marco del seminario de posgrado de Antropología de la Salud en FFyL-UBA. Agradezco a las profesoras Susana Margulies y M. Laura Recoder por el dictado de dicho seminario, a mis directoras de tesis, María Pozzio y Rosana Guber, y a mi directora de beca doctoral, Natalia Luxardo.

\section{Bibliografía}

Alonso, J. P. (2009). El cuerpo hipervigilado: incertidumbre y corporalidad en la experiencia de la enfermedad en Cuidados Paliativos. Cuadernos de Antropología Social, 29, 103-120.

Alonso, J. P. (2013). Cuidados paliativos: entre la humanización y la medicalización del final de la vida. Ciência \& Saúde Coletiva, 18 (9), 2541-2548.

Cantore, A. (2014). Antropología y la desnaturalización del sistema de salud: sobre la concepción de enfermedad de comunidades Mbyá en Misiones, En Letra, 1, diciembre: 142-163.

Cebolla Badie, M.; Dell'arciprete, A.; Fontes, C.; Hirsch, S. y Orlando, M. (2013). Uso, circulación y significación de los medicamentos en comunidades pilagá, guaraní, mbya-guaraní, tapiete y toba. Revista Argentina de Salud Pública, 17(4), 13-23.

Clark, D. y Seymour, J. (1999) Reflections on palliative care. Buckingham: Open University Press.
Cuyul, A.; Rovetto, M. y Specogna, M. (2011). Pueblos indígenas y sistemas de información en salud: la variable étnica en seis provincias argentinas. Revista Argentina de Salud Pública, 2 (7), junio: 12-18.

Del Monaco, R. (2013). Autocuidado, adherencia e incertidumbre: tratamentos biomédicos y experiencias de pacientes en el dolor crónico de la migraña. Salud Colectiva, 9(1):65-78.

Diehl, E.; Grassi, F. (2010). Uso de medicamentos em uma aldeia Guaraní do litoral de Santa Catarina, Brasil. Cadernos de Saúde Pública, 26 (8): 1549-1560

Dominguez Mon, A. (2015). Cuidados de sí y relaciones de género: trabajo en red en un grupo de personas que viven con diabetes (PVD) en un centro de atención primaria de la salud (CAP) en José León Suárez, Argentina. Política \& Trabalho, 42, 133-154.

Drovetta, R. (2009). Biomedicina y políticas de salud reproductiva en un contexto rural indígena de la Puna jujena, Argentina. Barbaroi, 31, agostodiciembre:139-154.

Etkin, N. (1992). "Side effects": Cultural constructions and reinterpretations of Western Medicine. Medical Anthropology Quarterly, 6 (2): 99-113.

Hardon, A. (1987). The use of modern pharmaceuticals in a Filipino Village: doctor's prescription and self medication. Social Science and Medicine, 25(3): 277-292.

Heredia, C. (2017) Sentir dolor. Recorridos, búsquedas y terapias en Cuidados Paliativos Pediátricos (Tesis de maestría). IDES/IDAES-UNSaM, Buenos Aires.

Hirsch, S. y Lorenzetti, M. (Eds.). (2016). Salud pública y pueblos indígenas en la Argentina: encuentros, tensiones $e$ interculturalidad. Buenos Aires: UNSaM edita.

Illich, I. (1975). Nemesis Médica. La expropiación de la salud. Barcelona: Ed. Barral

Kamat, V. y Nichter, M. (1998). Pharmacies, selfmedication and pharmaceutical marketing in Bombay, India. Social Science and Medicine, 47 (6): 779-794.

Kottow, M. y Bustos, R. (2005). Antropología médica. Buenos Aires: Editorial Mediterraneo.

Lagndon, J.E. (1988). Saúde Indígena: A Lógica do Processo de Tratamento, Revista de Saúde em Debate, Janeiro: 12-15.

Lopes, N. (2001). Automedicação: algumas reflexões sociológicas. Sociologia, problemas e Práticas, 37 : 141-165. 
Lorenzetti, M. (2012). La dimensión política de la salud: las prácticas sanitarias desde las comunidades periurbanas wichí del departamento de San Martín (Salta). Publicar-En Antropología y Ciencias Sociales, X (XII), junio: 65-85.

Menéndez, E. (2003) Modelos de atención de los padecimientos: de exclusiones teóricas y articulaciones prácticas. Ciencia \& Saúde Coletiva, 8(1), 185-207.

Menéndez, E. (1994) Le enfermedad y la curación. ¿Qué es medicina tradicional? Alteridades, 4(7), 71-83.

Menezes Aisengart, R. A. (2004). Em busca da boa morte: Antropologia dos cuidados paliativos. Rio de Janeiro: Fiocruz.

Nichter, M. (2008). Global Health: Why Cultural Perceptions, Social Representations, and Biopolitics Matter. Arizona: University of Arizona Press.

Oliveira, R. C. (2012). Corpo, gênero, adolescência: discursos e (re)significações a partir da anorexia e da obesidade. Revista Latinoamericana de Estudios sobre Cuerpos, Emociones y Sociedad, 4 (9), 17-27.

Persson, A. (2004). Incorporating Pharmakon: HIV, Medicine, and Body Shape Change. Body \& Society, 10 (4), 45-67. https://doi.org/10.1177/1357034X04047855

Petryna, Adriana; Kleinman, Arthur y Lakoff, Andrew. (2006). Global Pharmaceuticals: Ethics, Markets, Practices. Duke University Press.

Reyes Pérez, Martín. (2011). Sujeto y corporalidad: la experiencia del padecimiento em contextos de hospitalización (Tesis doctoral). CIESAS, Guadalajara, México.
Rohden, Fabíola. (2018). "Hormones save you from everything": production of subjectivities and bodily transformations with the use of biomedical resources". Mana, 24(1): 199-229 - DOI http://dx.doi. org/10.1590/1678-49442018v24n1p199.

Rose, Nicolas. (2012). Políticas de la vida: biomedicina, poder y subjetividad en el siglo XXI. La Plata: UNIPE.

Salas Arrambide M. (2004). Los cuidados paliativos: un modelo de atención integral al niño gravemente enfermo y su familia. Anales de Pediatría, 61 (4), 330-335.

Van Der Geest Sjaak; Whyte, Susan Reynolds y Hardon, Anita. (2002). Social Lives of Medicines. New York: Cambridge University Press.

Van Der Geest, Sjaak y Whyte, Susan Reynolds. (2011). O encanto dos medicamentos: metáforas e metonímias. Soc. e Cult., Goiânia, 14 (2): 457-472.

Vega, Fanny. (2016). Terapéutica racional en atención primaria de la salud: enfermedades oncológicas, dolor y cuidados paliativos en el primer nivel de atención. Ciudad Autónoma de Buenos Aires: Ministerio de Salud de la Nación. REMEDIAR.

Williams, Simon; Martin, Paul y Gabe, Jonathan. (2011). Evolving sociological analyses of 'pharmaceuticalisation': a reply to Abraham. Sociology of Health \& IIIness, 33 (5): 729-730.

World Health Organization. (2002). National cancer control programmes: policies and managerial guidelines. Ginebra: WHO. 